\title{
IoT Based Irrigation Monitoring System
}

\author{
K. Sujatha, V. Srividhya, Bikrant Nayak \\ ${ }^{I}$ Professor, EEE Dept., Center for Electronics, Automation \& Industrial Research (CEAIR), \\ Dr. MGR Educational \& Research Institute, Maduravoyal, Chennai. \\ ${ }^{2}$ Assistant Professor, EEE Dept., Meenakshi College of Engineering, Virugambakkam, Chennai \\ ${ }^{3}$ Assistant Professor, EEE Dept., Dr. MGR Educational \& Research Institute, Maduravoyal, Chennai
}

\begin{abstract}
Farmers have been growing their crops and vegetables with varied success which depends on the moisture content of the soil. The major task is to monitor the moisture content of the soil and the other related parameters like temperature, humidity and light intensity so as to provide a closed loop monitoring system. So it is decided to monitor and automate the irrigation system for the advancement in agriculture field. By implementing this cost effective agriculture monitoring scheme a milestone can be created in green revolution. This module serves the purpose of monitoring the plants from anywhere at any point of time, by integrating this system with cloud technology.
\end{abstract}

Keywords - Internet of Things; Automatic Irrigation System; Raspberry Pi; Soil Moisture; Cloud Computing.

\section{Introduction}

An artificial method to supply water to the fields is called as Irrigation. It is usually used in inadequate rainfall period to assist the growing of crops in dry areas. Now days, water shortage is becoming one of the biggest problem in the world $[1,2]$.

\subsection{Types of Irrigation Systems}

There are many techniques shown in Figure 1 to save or to control wastage of water from agriculture. Different types of irrigation are used for management of water in agricultural land [3].

\subsubsection{Ditch Irrigation}

In this scheme the channels are formed in which the seedlings are planted. This system of irrigation was once very popular in the USA, but most have been replaced with modern systems [4].

\subsubsection{Terraced Irrigation}

This method is tedious because the cultivation fields are partitioned into steps through which the seedlings are planted. The water is allowed to flow through each step and this type is suitable in hilly areas [5].

\subsubsection{Sprinkler System}

Sprayer is used to spray water to agricultural lands from overhead sprayers. There is another scheme where the sprayers are placed underneath the ground to irrigate the fields [6].

\subsubsection{Rotary Systems}

In this scheme the water sprayer is fitted with a motor to change its orientation, so that, it can spray water to the fields for irrigation. This system waters a larger area with small amounts of water over a longer period of time [7].

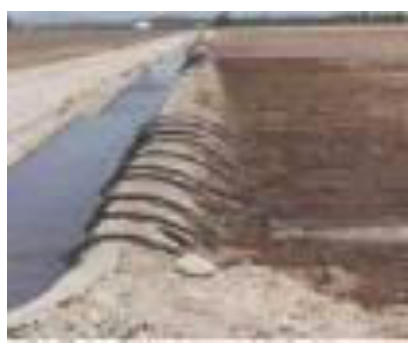

(a): Ditch Irrigation

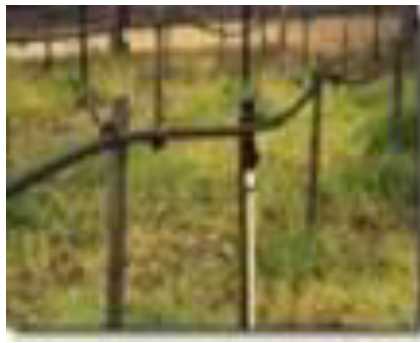

(c): Sprinkler System

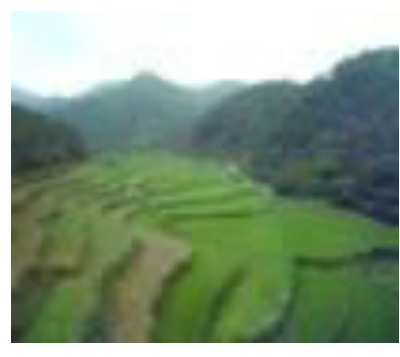

(b): Terraced Irrigation

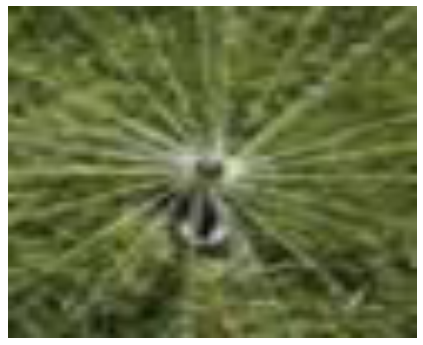

(d): Rotary Systems
Figure 1 (a, b, c, d): Types of Irrigation System

\section{Need for Automatic Irrigation}

The flexible form of irrigation is the Automatic irrigation systems. The automatic irrigation systems can used to save money and water management. Dead lawn 
grass and plants are to be replaced as if very expensive. Even though, the savings from automatic irrigation systems is more effective. Watering with a hose or oscillator waste water are not targets roots of the plant with significant degree of precision. But, automatic irrigation systems can be programmed to release more precise amounts of water in a targeted area, which promotes water conservation [8].

\section{Related Work}

In India, widely used irrigation techniques are manually operated due to its less expense and high profits. So, automatic irrigation techniques are not so common here. Nowadays, time controlled automatic sprayers are used for gardening at houses. The time interval is calculated by the volume of water to be supplied and the rate of flow of water $[9,10]$.

If the water in the tray is reached up to the mark of position, the sensor complete the circuitry and starts to isolate irrigation system from the supply and thus avoid excessive water supply. This method is patent to Richard E. Morrison, (Salt Lake City, UT) and Kent C. Erickson (Centerville, UT) [11]. In addition, they may require frequent resetting to achieve high levels of irrigation efficiency [12].

\section{Objective of the work}

The researchers are emerging a type of concealed irrigation system. The main disadvantage is that the water evaporates on exposure to atmosphere. Hence different types of sensors are buried under the ground to supply water to the crops. Such a scheme would reduce the usage of water for irrigation [13].

\section{Methodology}

Arduino programming for Intel Galileo Gen2 is done to record the sensor values. Sensors are used for health care data collection. Interfacing with physical world such as digital sensors, GPRS/GSM, and I2C is possible with Intel Galileo Gen2, and hence it is chosen for implementation. Intel Galileo Gen2 also offers the connectivity of sensors through cloud with the Wi-Fi support through miniPCIe slot available; leading to IoT based health care system [14]. Intel provides end to end IoT Solutions so that one could quickly connect, manage, and protect the digital sensors. [15].

- Rx of hardware serial port is connected to Galileo's D0.

- Tx of hardware serial port is connected to Galileo's D1.

- $5 \mathrm{~V}$ of hardware serial port is connected to Galileo's $5 \mathrm{~V}$.

- GND of hardware serial port is connected to Galileo's GND.

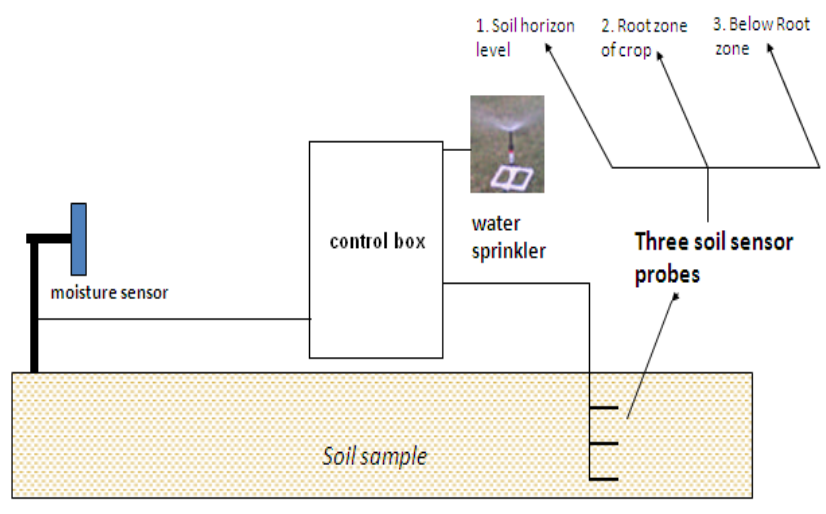

Fig. 2: Moisture Sensor Technology

\section{Results and Discussion}

The soil moisture monitoring is accomplished by making inserting the sensor into the soil, and it starts transmitting the moisture level corresponding to low, medium and high. This is deployed in Thingspeak cloud through IoT agent. From the cloud the information goes to the monitoring interface. From the interface the mobile phone could be connected. Alternatively the IoT agent is connected to the mobile phone with SMT package and SIM card inserted to it as shown in Figure 4, 5 and 6 respectively. The alert system is based on threshold calculated. The proposed system is designed to send alert message instantly, once the moisture level in the soil is detected. The system has also the ability to notify the conditions whether the soil is watered or dry. The alert messages are generated at mobile phone. Extracted parameters of the soil give the alert signals after comparison with assigned threshold [16] values. These alert signals indicate the presence or absence of the moisture in the soil.

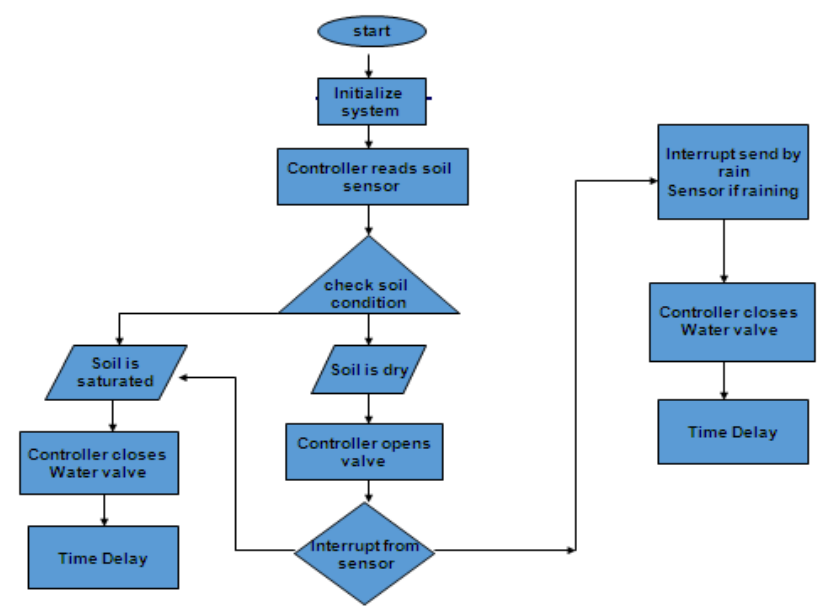

Fig.3: Flow chart of the sensing the Moisture level in soil

Figure 7 and 8 shows the deployment in Thingspeak cloud for measuring moderate moisture level in soil. Table 
I shows the values of the moisture level for low, medium and high conditions. It is compared with the existing system [16], and it is found to provide the alert with lesser response time, and hence the performance of the proposed system is improved as for as the heart beat rate monitoring is concerned. Figure 9 indicates the measurement of various parameters like humidity, soil moisture level, temperature and light intensity which inturn affects the growth of the crops.

\subsection{Digital Sensor}

A digital sensor interacts with the physical environment and returns binary information like a digital push button is pressed, or not.

It has two states, 0 or 1 . Three wires can be connected:

- A black one, for the ground.

- A red one, for input voltage.

A green one, transmitting the information.

- This moisture sensor measures the water present in the soil.

- This sensor has two probes, one is to pass current through the soil and another is to read the resistance to get the moisture level.

\subsubsection{Connect the Sensor to the AO(Analog 0) Pin on the Arduino Board}

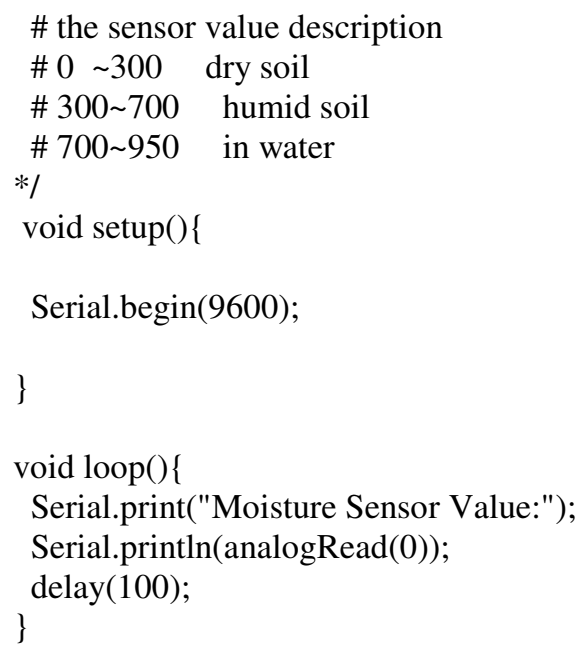

\subsection{Steps to Perform while Writing Test Case}

- Initialize pins (Digital and analog)

- Set the mode of particular pins only (relevant for digital)

- Read the Sensor Value

- Convert the value to voltage

- Display both sensor values and corresponding analog values on serial monitor
- Depending upon sensor values actuate the output device.

\subsection{Pseducode}

void setup() \{

// put your setup code here, to run once

Serial.begin $(9600)$; \}

void loop() \{

// put your main code here, to run repeatedly

Serial.print("moisture sensor value");

Serial.println(analogRead(1)); delay(100);

1
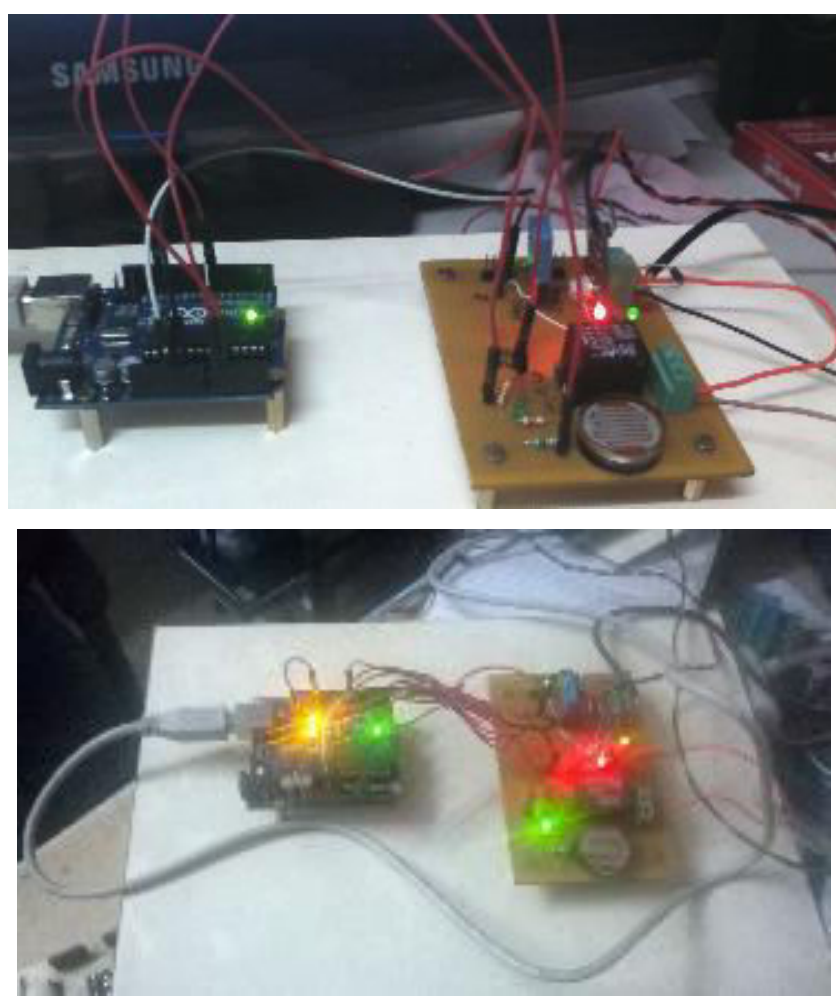

Fig.4: SIM Card inserted to Galileo Gen2

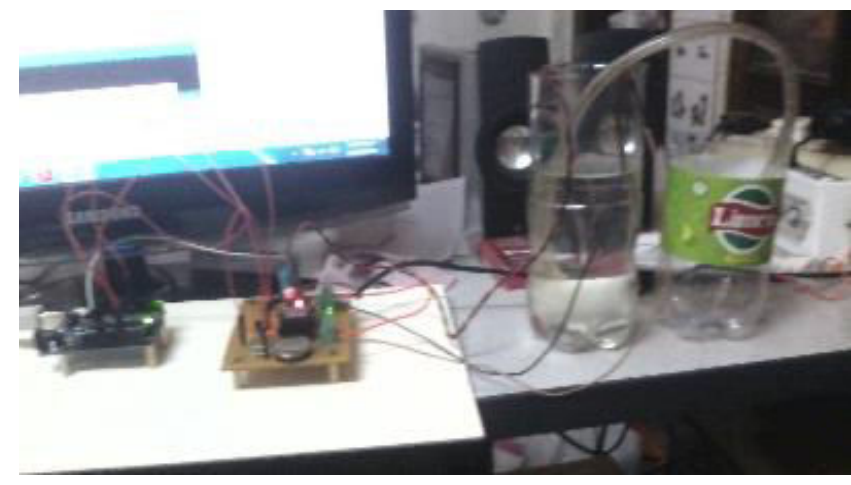

Fig.5: Moisture sensor with Intel Galileo Gen2

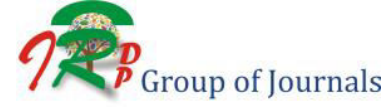




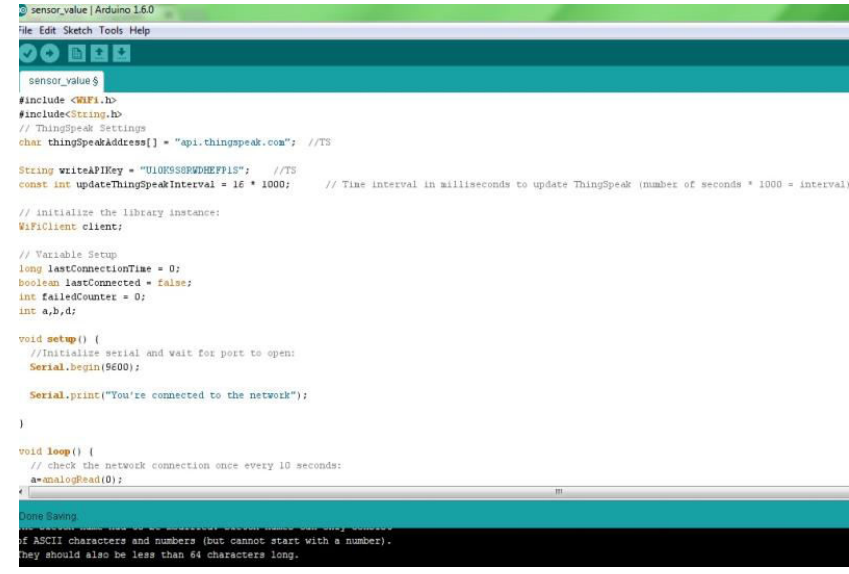

Fig.6: Enabling WiFi services using Intel Galileo Gen2

EAdd Visualizations

Data Export

\section{Channel Stats}

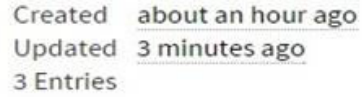

\section{Field 1 Chart}

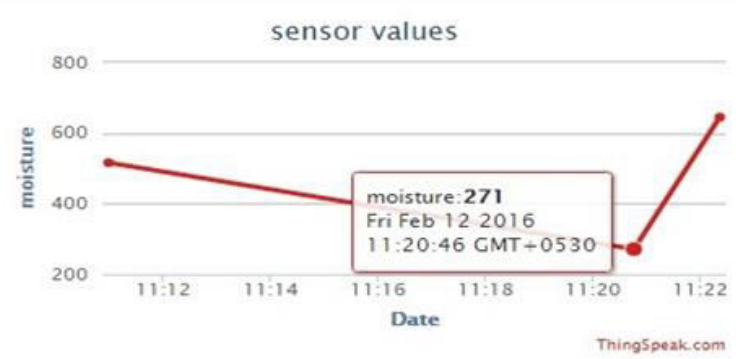

Fig.7: Deployment in Thingspeak cloud for measuring moderate moisture level in soil

\subsection{SMS Service for Moisture Level}

\#include <String.h>

void $\operatorname{setup}()$

\{

Seriall.begin(115200);

Serial.begin(9600);

delay(1000);

\}

void loop()

\{

if (Serial.available())

switch(Serial.read())

\{

case 't':SendTextMessage();

break;

case ' $r$ ':ReceiveTextMessage(); break;

case 'd':DialVoiceCall();

break;

case 'a':AnswerVoiceCall();

break;

case 'g':GPRS();

break;

\}

if (Serial1 available ())

Serial.write(Serial1.read());

\}

void AnswerVoiceCall()

\{

Serial1.println("ATA");

\}

void SendTextMessage()

\{

Serial1.print("AT+CMGF=1 $\backslash r ")$;

SMS in text mode

delay(1000);

Serial1.println("AT+CMGS=|"+917829374149\""); delay(1000);

Serial1.println("Hello from Galileo?"); delay(1000);

Serial1.println((char)26); //the ASCII code of the ctrl+z is 26 (0x1A)

delay(1000);

Serial1.println();

\}

void ReceiveTextMessage()

\{

Serial1.println("AT+CMGF=1"); $\quad$ // To receive the SMS in text mode

delay(1000);

Serial1.println("AT+CPMS=।"SM $\ "$ "); // read first SMS

Serial.println("Connection to ThingSpeak failed

("+String(failedCounter, DEC)+")");

Serial.println();

\}

\}

else

\{

failedCounter++;

Serial.println("Connection to ThingSpeak Failed

("+String(failedCounter, DEC)+")");

Serial.println();

lastConnectionTime $=$ millis () ;

\}

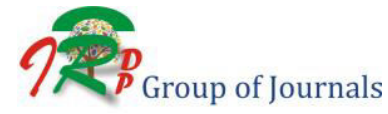


Table 1. Soil moisture level Measurement

\begin{tabular}{|c|c|c|}
\hline S.No & Moisture level category & Moisture level value \\
\hline 1. & Low & 23 \\
\hline 2. & Medium & 271 \\
\hline 3. & High & 645 \\
\hline
\end{tabular}

\section{$\square$ ThingSpeak Channels - Apps Blog Support -}

Private View Public View Channel Settings API Keys Data Import/Export

GAdd Visualizations Data Export

Channel Stats

Created about an hour ago
Updated 6 minutes ago

3 Entries

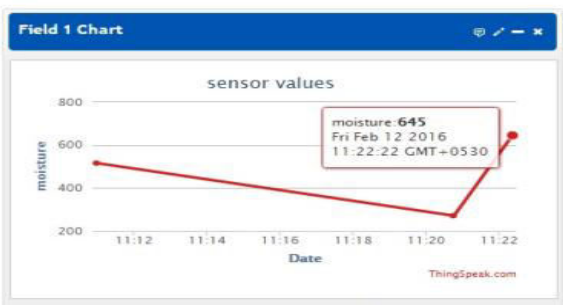

Fig.8: Deployment in Thingspeak cloud for measuring High moisture level in soil
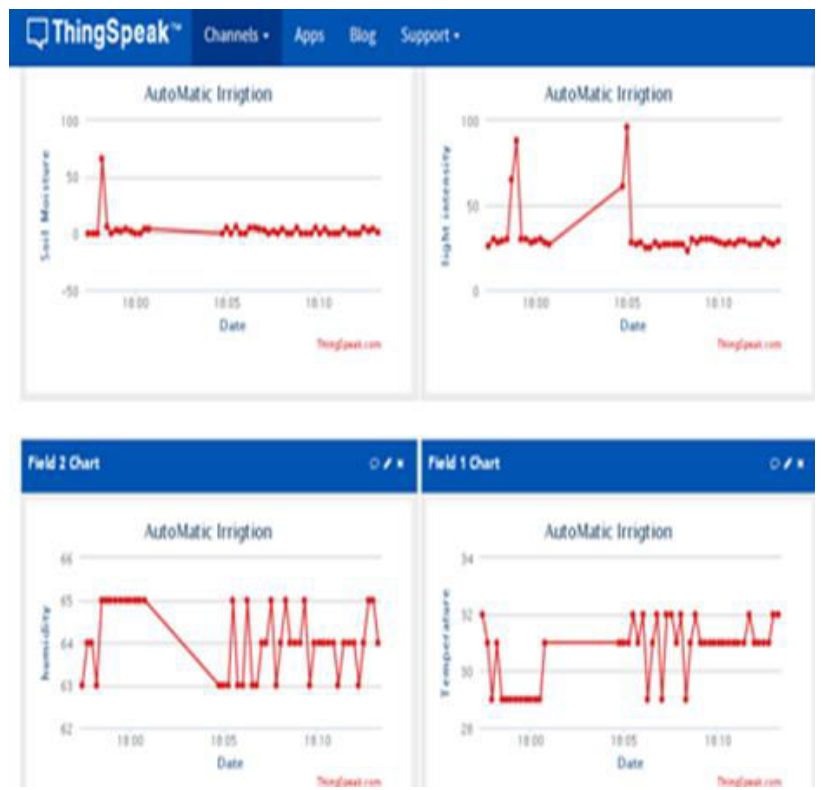

Fig.9: Deployment in Thingspeak cloud for measuring Soil mositure level, humidity, temperature and light intensity

\section{Conclusion}

Thus the Automatic Irrigation System has been successfully designed and tested. It has been developed by integrating the features of all used hardware components. All used modules are reasoned out and placed carefully to contribute the best working of the unit. Thus monitored the function of the motor automatically using GPRS technology got designed with the specific parameters. Then, highly advanced IC's are used with the help of growing technology and thus the project has been successfully implemented. This will be an efficient technique to control the motor functioning. The on and off action of the motor is controlled by using Microcontroller. It will have a demand in the places where the power fluctuations are considerably high.

\section{References}

[1] https://www.healthtap.com/topics/symptoms-usually-affect-fingerstoes-nose-lips-or-earlobes

[2] http://patient.info/health/raynauds-phenomenon-leaflet

[3] Sebastian Zickau, Dirk Thatmann, Tatiana Ermakova,Jonas Repschl"ager, R"udiger Zarnekow, and Axel K"upper, "IEEE 3rd International Conference on Cloud Networking"pp.333-338, 2014.

[4] Nimmy John, Sanath Shenoy, "Health Cloud - Healthcare As A service(HaaS)",International Conference on Advances in Computing,Communications and Informatics (ICACCI) , pp.19631966, 2014.

[5] Zhiyuan Lu, Xiang Chen, Zhongfei Dong, Zhangyan Zhao, Xu Zhang, "A Prototype of Reflection Pulse Oximeter designed for mobile health care, DOI 10.1109/JBHI.2015.2465861, IEEE Journal of Biomedical and Health Informatics, No. of pages 12, 2015.

[6] Andreas K. Triantafyllidis, Carmelo Velardo, Dario Salvi, Syed Ahmar Shah, Vassilis G. Koutkias, and Lionel Tarassenko, A Survey of Mobile Phone Sensing, Self-reporting and Social Sharing for Pervasive Healthcare,DOI 10.1109/JBHI.2015.2483902, IEEE Journal of Biomedical and Health Informatics, 2015.

[7] S. M. Riazul Islam, Daehan Kwak ; MD. Humaun Kabir ; Mahmud Hossain, "The Internet of Things for Health Care: A Comprehensive Survey", IEEE Access (Volume:3 ), pp. 678 - 708,2015.

[8] John Glaser, Devices everywhere talking to devices anywhere could radically change health care.June $4,2015$.

[9] Dmitry G. Korzun, Ilya Nikolavskiy, and Andrei Gurtov, "Service Intelligence support for medical sensor networks in personalized mobile health systems" Internet of things, smart spaces, and next generation networks and systems, LNCS 9247, Springer.

[10] G. Terrasson, Alvaro Llaria , R. Briand, System level dimensioning of low power biomedical Body Sensor Networks", IEEE Faible Tension Faible Consommation (FTFC), 10.1109/FTFC.2014.6828621, pp.1-4,2014.

[11] Alexandros Pantelopoulos,Nikolaos G. Bourbakis, "A survey on wearable sensor-based systems for health monitoring and prognosis" ,IEEE Transactions on Systems, Man, and Cybernetics, Part C Applications and Reviews, Vol. 40 Issue 1, Pages 1-12, 2010.

[12] How the Internet of Things is changing healthcare and transportation http://www.cio.com/article/2981481/healthcare/how-the-internet-ofthings-is-changing-healthcare-and-transportation.html.

[13] Luca Catarinucci, Lecce, Italy , Danilo De Donno , Luca Mainetti , Luca Palano "An IoT-Aware Architecture for Smart Healthcare Systems" IEEE Internet of Things Journal (Volume:2 , Issue: 6 ), pp..515 - 526, 2015.

[14] IoT Healthcare Solutions Kaa - a leading IoT platform for state-ofthe-art Healthcare, http://www.kaaproject.org/healthcare/

[15] David Niewolny, "How the Internet of Things Is Revolutionizing Healthcare" ,White paper.

[16] Priyanka Kakria, N. K. Tripathi,and Peerapong Kitipawang," A Real-Time Health Monitoring System for Remote Cardiac Patients Using Smartphone and Wearable Sensors" ,International Journal of Telemedicine and Applications Vol. 2015 (2015), Article ID 373474, 11 pages, http://dx.doi.org/10.1155/2015/373474, 2015.

[17] https://www.openhacks.com/page/productos/id/546/title/ATWINQuad-band-GPRS-GSM-Shield-for-Arduino 